\section{Os Suplementos Saúde na Pesquisa Nacional por Amostra de Domicílios (PNAD) no Brasil}

\author{
Health supplements in the Brazilian \\ National Household Survey - PNAD
}

\section{Resumo}

As mudanças socioeconômicas, demográficas e tecnológicas e suas implicações nas políticas públicas demandam dos órgãos governamentais a produção de informações. As informações atualizadas de base populacional e de âmbito nacional são essenciais ao processo de planejamento e ao acompanhamento pela sociedade do cumprimento dos princípios constitucionais da saúde, como direito ao acesso igualitário aos serviços de saúde. A Pesquisa Nacional por Amostra de Domicílios (PNAD) é uma fonte de dados importante para o conhecimento e monitoramento de aspectos relevantes da situação de saúde da população brasileira e do acesso, utilização e financiamento de serviços de saúde. Neste artigo, faz-se um breve histórico da evolução dos objetivos, da periodicidade e da abrangência geográfica ao longo de quatro décadas da PNAD no Brasil, enfatizando-se os principais aspectos incluídos nos suplementos de saúde em 1981, 1986, 1998 e 2003. A produção de textos técnicos e acadêmicos, gerados a partir desses suplementos, tem permitido conhecer aspectos importantes da saúde da população brasileira e monitorar, em diferentes recortes geográficos e socioeconômicos, as políticas voltadas para o acesso e uso de serviços de saúde. Argumenta-se sobre a necessidade de iniciar uma discussão mais profunda sobre a continuidade da série histórica iniciada em 1998, frente à implantação, em futuro próximo, do Sistema Integrado de Pesquisas Domiciliares (SIPD) pelo IBGE.

Palavras-chave: Inquéritos domiciliares. PNAD. Saúde. Acesso a serviços de saúde. Utilização de serviços de saúde.

\section{Claudia Travassos \\ Francisco Viacava \\ Josué Laguardia}

Instituto de Comunicação e Informação Científica e Tecnológica em Saúde FIOCRUZ, RJ

Correspondência: Claudia Travassos, Avenida Brasil, 4365, Biblioteca de Manguinhos, $2^{\circ}$ andar, Manguinhos - Rio de Janeiro, RJ, CEP 21045-900,E-mail: claudia@cict.fiocruz.br 


\section{Abstract}

Socioeconomic, demographic and technological changes as well as their implications for public policies call for the production by governmental institutions for comparable and updated information. Population-based information at the national level is essential for planning and monitoring health policies and their constitutional principles, such as universal and equitable access to healthcare. The Brazilian National Household Survey (PNAD) is a relevant data source for the knowledge and monitoring of important aspects of health status as well as of the policies directed toward the access and use of health services. In this paper, we present a brief history of the objectives, frequency and geographical coverage of PNAD in Brazil over the past four decades. Emphasis is placed on the main contents of its Health Supplements in 1981, 1986, 1998 and 2003. The publication of technical and academic texts based on these data shows that they can be used to monitor access and utilization of health services in different geographical and socioeconomic contexts. We argue in favor of an in-depth discussion on the continuity of the historical series initiated in 1998, in face of the implementation in the near future of the Household Surveys Integrated System (SIPD) by IBGE.

Keywords: Household Surveys Integrated System. PNAD. Health. Access to health services. Health services utilization.

\section{Breve histórico da Pesquisa Nacional por Amostra de Domicílio - PNAD}

No início da década de 1960, a United States Agency for International Development (USAID) e o Inter-American Statistical Institute (IASI) propuseram, a partir das experiências estadunidense e canadense com pesquisas contínuas de população, a realização de inquéritos para os países da América Latina, de modo a subsidiar o planejamento do desenvolvimento socioeconômico ${ }^{1}$.

Essa proposta, conhecida como "Plano Atlântida”, foi apresentada em 1962, no México, em 14 volumes, que detalhavam todo o desenvolvimento das pesquisas desde o seu planejamento até a execução e a análise dos dados ${ }^{2}$. Nela, as pesquisas eram contínuas e iriam a campo trimestralmente, com amostras de âmbito nacional de cerca de 10 mil domicílios. O objetivo principal era gerar informações sobre as características demográficas básicas da população e sobre o mercado de trabalho. As entrevistas tinham a duração de 15 a 20 minutos. A proposta contemplava a inclusão, no questionário básico, de temas suplementares, com periodicidade variável segundo a natureza do tema.

No Brasil, a primeira pesquisa foi aplicada no segundo trimestre de 1967, com uma amostra de cerca de 30 mil domicílios, cobrindo a área que atualmente corresponde ao Estado do Rio de Janeiro. Nos dois anos seguintes, foi realizada trimestralmente. Sua abrangência geográfica expandiu-se, no final da década de 1960, para as regiões Nordeste, Sudeste e Sul, e para o Distrito Federal. Em 1970, por ocasião da realização do censo demográfico, a pesquisa não foi realizada.

Em 1971, a PNAD foi retomada com modificações na periodicidade e no plano amostral. A coleta de dados deixou de ser contínua e passou a ser realizada uma vez por ano, no último trimestre. Entre 1973 e 1975, dada a realização do Estudo Nacional da Despesa Familiar (ENDEF), a PNAD deixou de ser aplicada. A partir de 1976, ela 
foi novamente retomada no mesmo formato de 1971. Por demanda de instituições governamentais responsáveis pelo planejamento regional, a amostra foi ampliada para as demais regiões, de forma a gerar dados representativos dos âmbitos nacional, estadual e das nove regiões metropolitanas existentes à época. Devido a dificuldades operacionais, não incluiu domicílios localizados no estrato rural da região Norte. A abrangência geográfica da amostra permaneceu com esse formato até 2004, quando passou a cobrir todo o território nacional.

A amostra da PNAD é selecionada em três estágios sucessivos: Municípios, Setores e Unidades Domiciliares. Existem municípios auto-representados e municípios escolhidos aleatoriamente. Os setores censitários, que constituem as unidades de seleção no segundo estágio, são áreas menores em que se subdividem os distritos e municípios para efeitos de levantamentos estatísticos por parte do IBGE, como os censos. Anualmente, são investigados na PNAD cerca de 110.000 domicílios.

\section{Estrutura do questionário do corpo básico da PNAD}

A estrutura da pesquisa contempla dois níveis de informação. No primeiro, incluemse dados relativos aos domicílios e, no segundo nível, dados sobre os indivíduos residentes, inclusive aqueles ausentes por um período não superior a doze meses em relação à data da entrevista. São pesquisados os domicílios particulares e as unidades de moradia (quarto, apartamento etc.) em domicílios coletivos. Os moradores de domicílios coletivos, “onde prevalece o cumprimento de normas administrativas" ${ }^{3}$, tais como hospitais, presídios e conventos, não são entrevistados. Em função da estrutura da pesquisa, a população moradora de rua não está incluída na amostra.

Atualmente, o questionário da PNAD contempla, no nível do domicílio, informações sobre características gerais, con- dição de ocupação, abastecimento de água, esgotamento sanitário, destino de lixo, iluminação elétrica, bens - que incluem telefones fixo e celular, microcomputador e acesso à internet - e características das famílias. A família é definida como "o conjunto de pessoas ligadas por laços de parentesco, dependência doméstica ou normas de convivência, que residem na mesma unidade domiciliar e, também, a pessoa que mora só em uma unidade domiciliar" ${ }^{3}$. Os dados individuais da PNAD permitem análises no âmbito da família.

No nível individual, o questionário contém informações demográficas, cor ou raça, migração, escolaridade, características do trabalho e rendimento para os maiores de 10 anos, características do trabalho infantil e características de fecundidade.

No que concerne aos suplementos temáticos, não houve um planejamento explícito sobre os temas e sua periodicidade, como previsto. Entretanto, alguns temas receberam maior destaque, tais como fecundidade (1972, 1973, 1978, e 1984) e, mais recentemente, saúde (1981, 1986, 1998 e 2003). Ao longo dos seus 40 anos de existência, alguns temas dos $\mathrm{Su}$ plementos foram incorporados ao corpo básico do questionário da PNAD, como foi o caso da fecundidade e do trabalho infantil (Quadro 1).

\section{O Suplemento Saúde da PNAD}

No caso específico da saúde, o primeiro suplemento foi a campo em 1981. O suplemento continha um amplo questionário que abordou diversos temas, incluindo morbidade, uso de serviços de saúde, cobertura vacinal, cuidado da mãe e da criança, hospitalização, assistência odontológica, portadores de deficiências e de incapacidade física. O suplemento também coletou dados sobre gastos privados em saúde e fontes de financiamento do consumo de serviços de saúde. Esse último permitiu identificar a participação dos principais subsetores de prestação e financiamento de serviços de 
Quadro 1 - Temas dos Suplementos da PNAD, 1972-2006.

Picture 1 - Themes of PNAD Supplements, 1972-2006.

\begin{tabular}{|c|c|}
\hline ANO & TEMA \\
\hline 1972 & Fecundidade, Rendimento, Bens de consumo \\
\hline 1973 & Fecundidade e Migração \\
\hline 1976 & Migração, Cor, Religião e Fecundidade \\
\hline 1977 & Bens de consumo duráveis, Características das habitações e Fecundidade \\
\hline 1978 & Fecundidade \\
\hline 1979 & Consumo de energia \\
\hline 1981 & Saúde \\
\hline 1982 & Educação \\
\hline 1983 & Previdência e Mão-de-obra \\
\hline 1984 & Fecundidade e Nupcialidade - transição demográfica \\
\hline 1985 & Situação do menor \\
\hline 1986 & Acesso a serviços de saúde, Suplementação alimentar, Associativismo, Anticoncepção \\
\hline 1987 & Cor (1 quesito) \\
\hline 1988 & Participação político-social/vitimização \\
\hline $1989-1990$ & Trabalho \\
\hline $1992-1993$ & Migração, Fecundidade e Nupcialidade \\
\hline 1995 & Migração, Fecundidade e Nupcialidade \\
\hline 1996 & Mobilidade social \\
\hline 1997 & Migração e Fecundidade \\
\hline 1998 & Acesso e utilização de serviços de saúde \\
\hline 1999 & Migração e Fecundidade \\
\hline 2001 & Trabalho infantil \\
\hline 2003 & Acesso e utilização de serviços de saúde \\
\hline 2004 & Educação, Segurança alimentar e transferência de renda de programas sociais \\
\hline 2005 & Acesso à internet e posse de telefone móvel celular para uso pessoal \\
\hline 2006 & $\begin{array}{l}\text { Trabalho infantil, aspectos complementares de educação e transferência de renda de programas } \\
\text { sociais }\end{array}$ \\
\hline 2007 & Educação de jovens e adultos e educação tecnológica profissional \\
\hline 2008 & Acesso e utilização de serviços de saúde \\
\hline
\end{tabular}

saúde à época - previdência social, sistema de pré-pagamento, sistema do empregador e recursos próprios.

Uma versão menor e diferente do suplemento de 1981 acompanhou a PNAD de
1986, que pesquisou aspectos relativos à morbidade referida, utilização de serviços, suplementação alimentar e anticoncepção. Em 1988, algumas questões sobre uso de medicamentos e uso de serviços públicos 
de saúde foram incorporadas ao Suplemento Participação Político-Social.

A formulação de alguns quesitos nos suplementos de 1981 e 1986 foi alvo de críticas. No suplemento de 1981, não houve independência entre as perguntas sobre morbidade, procura e uso de serviços de saúde, o que introduz erro nas análises sobre acesso e utilização de serviços de saúde. Por outro lado, as datas de referência para os quesitos sobre morbidade, procura e uso de serviços de saúde foram pré-fixadas, o que implicou em diferentes tempos de recordação, dependendo da data em que foi aplicada a entrevista, afetando a qualidade das respostas. No suplemento de 1986, esse problema foi corrigido, com os períodos de referência da pesquisa tendo como base a data da entrevista. Entretanto, manteve-se a dependência entre a pergunta de morbidade e as perguntas de procura e utilização (Quadro 2).

Somente em 1998, após um intervalo de 10 anos sem informação sobre saúde nos suplementos da PNAD, um novo suplemento sobre acesso e utilização de serviços foi aplicado. Com esse suplemento, inaugurase uma série histórica qüinqüenal de informação nacional de base populacional sobre saúde e consumo de serviços de saúde, financiada pelo Ministério da Saúde. Vale destacar que essa série de suplementos de saúde não foi planejada, mas resultou de oportunidades aproveitadas pelo Ministério da Saúde, que permitiram a aplicação do suplemento com pequenas modificações em 2003. A realização desses suplementos com intervalo de 5 anos estimulou a continuação da série com o compromisso do Ministro da Saúde, no momento da divulgação dos resultados do Suplemento 2003, de um novo suplemento de saúde em 2008.

O suplemento de 1998 passou a contar com seis blocos independentes, que foram respondidos por todas as pessoas: morbidade, cobertura por plano de saúde, acesso a serviços, utilização de serviços, limita-

Quadro 2 - Comparação entre os questionários dos Suplementos Saúde 1981, 1986 e 1998 em quesitos sobre morbidade referida, procura e uso de serviços de saúde.

Picture 2 - Comparison of the questionnaires of 1981, 1986 and 1998 Health Supplements on referred morbidity, search and utilization of healthcare services.

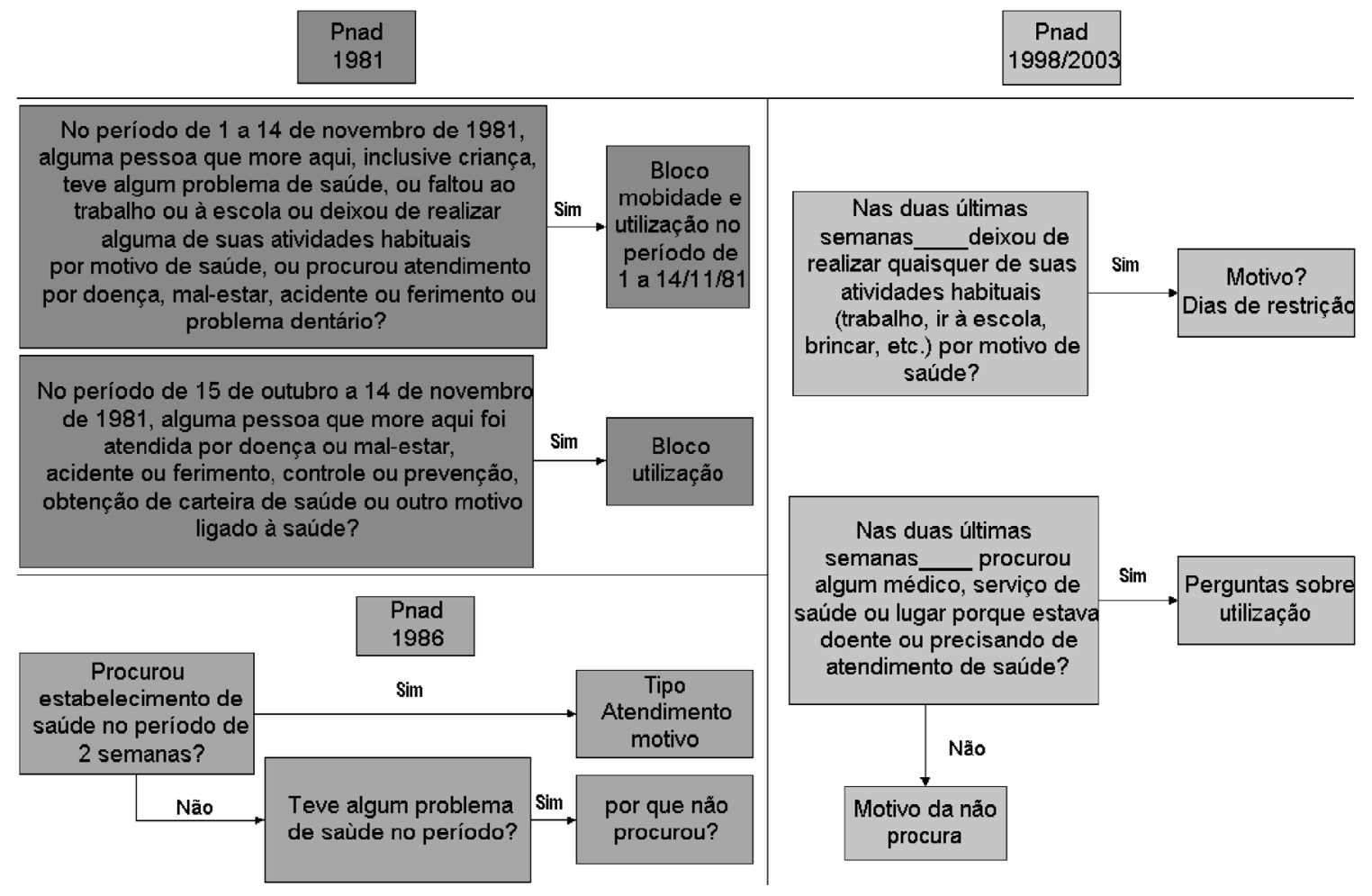


ção de atividades físicas para maiores de 13 anos e gastos privados com saúde.

No primeiro bloco do suplemento, existem quatro medidas de morbidade referida: auto-avaliação do estado de saúde, restrição de atividade por motivo de saúde, incapacidade funcional física e presença de doença crônica.

Diversos estudos têm demonstrado a importância da auto-avaliação como medida do estado geral de saúde. Essa medida mostra-se associada à mortalidade e ao uso de serviços de saúde ${ }^{4}$. A escala utilizada tem 5 graus, a exemplo da escala utilizada em outros inquéritos como o National Health Interview Survey, nos EUA; tanto, as categorias empregadas não são rigorosamente iguais. Uma limitação nos Suplementos Saúde da PNAD a ser destacada é a possibilidade de quesitos serem respondidos por outra pessoa, moradora ou não do domicílio. Questionários respondidos por substitutos (proxy) ocorrem com maior freqüência nos menores de 14 anos, cuja resposta é dada preferencialmente pela mãe. No suplemento de 1998, $51,3 \%$ dos maiores de 14 anos residentes nas áreas urbanas e $43 \%$ dos residentes nas áreas rurais não responderam pessoalmente o questionário. A alta freqüência de respostas fornecidas por informantes substitutos no suplemento da PNAD é decorrente da logística de coleta dos dados para contenção dos custos da pesquisa. Como não é prevista uma segunda visita do entrevistador, os dados dos moradores ausentes são fornecidos por aqueles que se encontram no domicílio no momento da entrevista. O emprego de informantes substitutos em pesquisas domiciliares é comumente adotado por órgãos estatísticos governamentais de diversos países. Estudos realizados com dados dos Suplementos Saúde têm demonstrado que há diferença nos resultados quando a autoavaliação é realizada por outra pessoa e recomendam que as análises sejam ajustadas pelo tipo de informante $\mathrm{e}^{6,7}$.

A restrição de atividades rotineiras por motivo de saúde refere-se a comportamen- tos geralmente associados à redução de atividades que a pessoa é capaz de desempenhar no seu dia a dia. Geralmente indica uma redução transitória nas atividades do indivíduo, abaixo de suas capacidades normais. É, assim, um indicador da ocorrência de problemas agudos de saúde.

As perguntas de incapacidade física funcional medem a condição dos indivíduos para realizar atividades rotineiras em função de suas condições de saúde. As perguntas dessa seção são baseadas no questionário do Jamaica Survey of Living Conditions, de 1989, e possibilitam medir graus variáveis de incapacidade em mobilidade física ${ }^{8}$.

Uma lista de 15 doenças crônicas foi selecionada a partir de um conjunto de critérios não excludentes: alta prevalência (exemplo: hipertensão), altas taxas de utilização de serviços de saúde (ex: bronquite e asma), possibilidade de intervenção efetiva (ex: tuberculose) e elevada freqüência de uso de serviços de alto custo (ex: insuficiência renal crônica, câncer). Foram incluídas também a tendinite e a tenosinovite, problemas cuja ocorrência tem crescido no país. A formulação do quesito referente às doenças crônicas foi alterada no suplemento de 2003. Em 1998, para cada uma das doenças crônicas perguntava-se "Sr/Sra tem ...?". Frente a uma possível superestimativa da prevalência de condições crônicas induzida por essa formulação, decidiu-se modificá-la para: “algum médico ou profissional de saúde disse que o Sr/Sra tem ...?". Como era esperado, a reformulação das perguntas levou à diminuição da prevalência de doenças crônicas. Como a resposta, em 2003, passou a refletir o acesso a um profissional de saúde e, dada a marcada desigualdade social existente no Brasil no acesso em favor dos mais ricos, verificou-se uma inversão no gradiente social da prevalência de doenças crônicas. Enquanto em 1998 as prevalências eram inversamente relacionadas à renda, em 2003 passou-se a observar uma associação positiva com a renda (Gráfico 1).

O segundo bloco do questionário trata do tema seguro de saúde e permite calcu- 


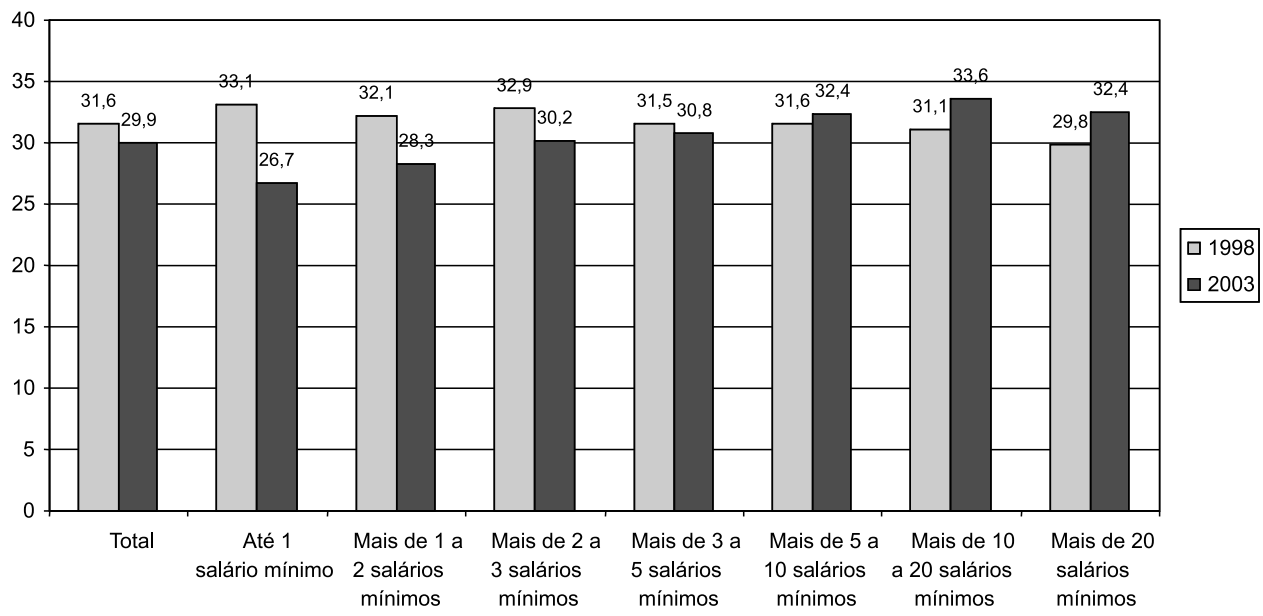

Gráfico 1 - Percentual de pessoas que referem pelo menos uma doença crônica segundo classe de renda familiar. Brasil, 1998 e 2003.

Graph 1 - Percentage of individuals who referred at least one chronic condition according to family income group. Brazil, 1998 and 2003.

lar a cobertura populacional por planos de saúde, que pode ser desagregada segundo planos privados e de instituições de assistência ao servidor público. Todos os entrevistados respondem à pergunta inicial do bloco sobre ter direito a um plano de saúde. Entretanto, as perguntas sobre as características dos planos são respondidas apenas pelo titular e as respostas são imputadas a todos os dependentes e agregados. Dessa forma, é possível analisar para todos os respondentes as características do plano, tais como os serviços cobertos pelo plano, a fonte de pagamento das mensalidades, a existência de co-pagamento, a possibilidade de reembolso e a satisfação do usuário. Como o titular do plano está identificado no questionário de todos os dependentes, é também possível realizar análise no nível dos contratos, isto é, descrever o número de pessoas por contrato segundo características sociodemográficas e de saúde. A cobertura por planos odontológicos modalidade que começa a ser oferecida pelas empresas - também pode ser monitorada.

O terceiro e quarto blocos permitem gerar indicadores de acesso e uso dos serviços de saúde pela população. Um indicador de acesso é obtido a partir da freqüência com que os indivíduos referem ter um serviço de saúde ao qual normalmente recorrem quando necessitam de atendimento. Há evidências na literatura sobre a importância do uso regular de um serviço e/ou profissional para uma adequada utilização dos serviços de saúde ${ }^{9}$. No suplemento de 2003, foram incorporados quesitos sobre o uso de procedimentos preventivos de câncer de mama e de útero em mulheres com idade igual ou superior a 25 anos, que são iguais aos quesitos do Inquérito Domiciliar sobre Comportamentos de Risco e Morbidade Referida de Doenças e Agravos não Transmissíveis, realizado pelo Instituto Nacional do Câncer (INCA) em $2003^{10}$.

No quarto bloco, é possível analisar a procura por serviços de saúde segundo o motivo e tipo de prestador. Aos que procuraram e não foram atendidos, pergunta-se o motivo. Aos que foram atendidos, é perguntado o tipo de atendimento recebido, a prescrição de medicamentos, a satisfação com o atendimento, as fontes de financiamento do atendimento, entre outros quesitos (Quadro 3). É possível obterse também a reincidência da procura para conseguir um atendimento. A reincidência da procura é uma outra dimensão de acesso aos serviços de saúde e busca medir com que freqüência (insistência) uma 
Quadro 3 - Fluxograma para utilização de serviços de saúde

Picture 3 - Flow chart of utilization of healthcare services

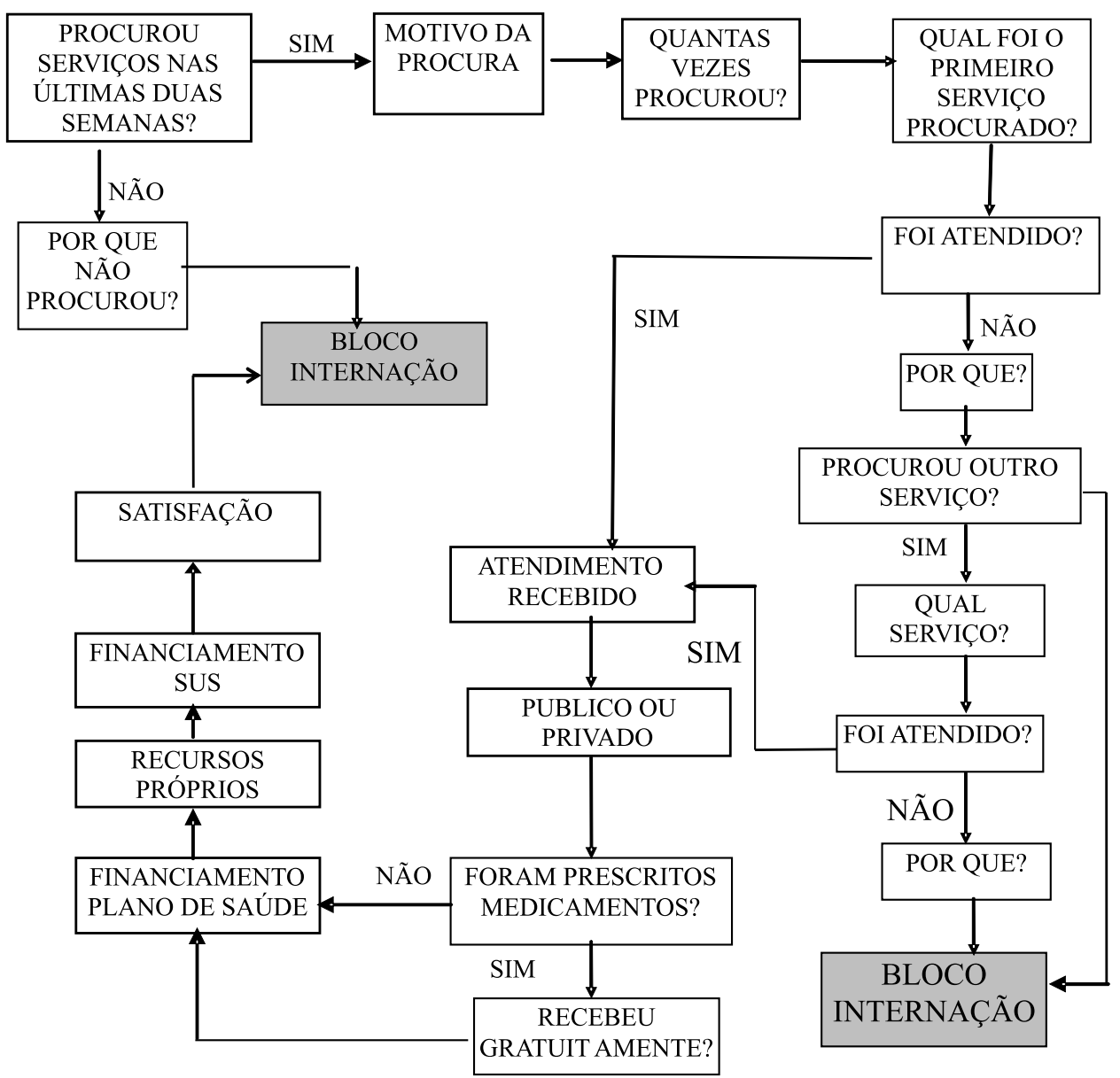

pessoa teve de procurar serviços para ser atendida no período de referência.

O uso de serviços hospitalares no ano que antecedeu a entrevista é investigado separadamente. Esses dados permitem conhecer a proporção de pessoas que se internaram no período, segundo a fonte de financiamento e o número de internações por pessoa no período, informação não disponível nos bancos de dados do Sistema de Informações Hospitalares do SUS SIH/SUS. Em acréscimo, as pesquisas domiciliares geram informações complementares às regularmente disponíveis nos bancos de dados administrativos, além de incluir dados sobre os indivíduos que não procuraram ou não conseguiram utilizar os serviços de saúde.

As perguntas sobre as fontes de finan- ciamento dos atendimentos à saúde permitem mensurar a participação do SUS, do gasto privado e do plano de saúde, e as possíveis combinações entre elas, de modo a caracterizar os diversos mercados de serviços de saúde no país. Permitem também medir a participação simultânea no financiamento do atendimento do plano de saúde e do gasto privado, que indica o emprego de estratégias de contenção de demanda pelos planos de saúde, como, por exemplo, o co-pagamento (Quadro 4).

O gasto privado em saúde, informação coletada apenas em 1998, gera informação sobre os gastos das famílias no consumo de bens, serviços de saúde e medicamentos. Esses dados podem ser correlacionados com o rendimento familiar, permitindo identificar o peso desse tipo de despesa no orça- 
Quadro 4 - Fontes de financiamento do uso de serviços de saude nos quinze dias que antecederam a entrevista, PNAD, 2003

Picture 4 - Funding sources for the use of healthcare services in the fifteen days before the interview, PNAD, 2003

\begin{tabular}{|c|c|c|c|c|}
\hline Fontes de Financiamento & Plano de Saúde & Gasto do Bolso & SUS & Outras Fontes \\
\hline \multirow[t]{2}{*}{ Plano de Saúde } & $\begin{array}{c}\text { Prêmios pagos a } \\
\text { Planos }\end{array}$ & $\begin{array}{c}\text { Plano } \\
\text { (ex:co-pagamento) }\end{array}$ & & \\
\hline & $23,3 \%$ & $3,3 \%$ & $0,3 \%$ & \\
\hline \multirow[t]{2}{*}{ Gasto do Bolso } & & $\begin{array}{l}\text { Gasto Privado } \\
\text { Direto }\end{array}$ & & \\
\hline & & $11,8 \%$ & $0,8 \%$ & \\
\hline \multirow[t]{2}{*}{ SUS } & & & Tributos & \\
\hline & & & $48,2 \%$ & \\
\hline Outras Fontes & & & & $\begin{array}{c}\text { Outras Fontes } \\
8,6 \%\end{array}$ \\
\hline
\end{tabular}

mento das famílias. A partir desses dados, pode-se também estimar a parcela correspondente aos recursos privados envolvidos no gasto total em saúde no país. Estudo realizado sobre o gasto privado em saúde captado pela PNAD e pela Pesquisa de Orçamento Familiar (POF) demonstrou que, apesar das diferenças nas metodologias de coleta, os resultados em termos relativos foram muito semelhantes nas duas pesquisas ${ }^{11}$. Entretanto, esse bloco foi retirado do questionário em 2003 pelo IBGE.

O questionário em elaboração, que irá a campo em 2008, manterá a estrutura dos Suplementos anteriores, mas deverá incluir novos temas sobre aspectos comportamentais relacionados à saúde, como tabagismo e sedentarismo/atividade física. Os quesitos relativos ao tabagismo correspondem às perguntas do Inquérito realizado pelo INCA em $2003^{10}$. Outros temas incluídos neste novo suplemento são: violência, uso de cinto de segurança, acidente de trânsito, atendimento de emergência no domicílio e cadastramento no programa Saúde da Família.

\section{Publicações geradas pelos Suplementos Saúde da PNAD}

Em revisão da literatura sobre relatóri- os técnicos e artigos científicos que empregaram na análise dados dos Suplementos Saúde da PNAD, encontraram-se 52 publicações até maio de 2007. A revisão foi realizada nas bases de dados LILACS, Scielo e Medline, utilizando-se as palavras-chave saúde (health) e PNAD. Aos textos encontrados nessas bases, foram incorporados mais quatro textos selecionados em pesquisa no Google, empregando-se as mesmas palavras-chave, e um relatório técnico elaborado pelo Ministério da Fazenda. Não foram incluídas na revisão teses e apresentações em congressos.

O Suplemento Saúde da PNAD 1981 gerou 6 publicações ${ }^{12-17}$, sendo que 5 são capítulos de um documento publicado pelo IBGE, em 1984, sobre a situação de saúde de crianças e mães no Brasil. Os temas analisados correspondem àqueles incluídos no suplemento, com destaque para a vacinação, assistência à gestação e ao parto, e aleitamento materno, eixo das políticas de saúde pública à época. A avaliação da saúde e da assistência maternoinfantil realizada por técnicos do IBGE teve como foco as desigualdades sociais e geográficas, tema que vai pautar grande parte dos futuros estudos com os dados dos Suplementos Saúde da PNAD. A outra publicação é um artigo ${ }^{17}$ da Revista Ciência e 
Cultura, da Sociedade Brasileira para o Progresso da Ciência - SBPC, que compara resultados de um inquérito realizado no estado da Bahia sobre morbidade e utilização de serviços de saúde, com os dados do Suplemento Saúde 1981, enfatizando nas desigualdades urbanas e rurais.

Por sua vez, o Suplemento Saúde da PNAD 1986 gerou duas publicações. A primeira correspondeu a um Relatório Técnico ${ }^{18}$, publicado pela Escola Nacional de Ciências Estatísticas - ENCE/IBGE, que analisou dados de morbidade e utilização de serviços de saúde sob a ótica das desigualdades sociais e geográficas, bem como da eficiência da rede de serviços de saúde. A segunda, um artigo científico ${ }^{19}$, concentrou sua análise no diferencial de gênero na morbidade e na utilização de serviços de saúde entre os Estados brasileiros.

Há um intervalo de 12 anos entre o Suplemento Saúde da PNAD 1986 e 1998, no qual nenhuma outra publicação com dados dos Suplementos Saúde foi encontrada.

Com a divulgação dos microdados do Suplemento Saúde 1998, houve um aumento expressivo de publicações. Até maio de 2007, são 33 publicações em revistas científicas nacionais e internacionais, publicações por universidades e órgãos de governo, que analisaram dados do Suplemento. Do total, apenas duas publicações são relatórios técnicos de órgãos de governo, uma do IPEA ${ }^{20}$ e outra do Ministério da Fazenda $^{21}$. Comparativamente ao observado com o Suplemento Saúde de 1981, em que a maioria das publicações é oriunda do próprio IBGE, há um importante incremento da produção acadêmica baseada nos dados do Suplemento Saúde da PNAD. Em parte, esse aumento foi estimulado pela produção de um suplemento temático na revista Saúde \& Ciência Coletiva, em 2002.

Os microdados do Suplemento Saúde 2003 foram divulgados pelo IBGE em 2004. Até maio de 2007, foram encontradas 12 publicações em revistas científicas que analisaram dados do Suplemento 2003; dessas, a metade são comparações com os resultados observados em 1998. Um novo suplemento temático da Saúde \& Ciência Coletiva foi responsável pela quase totalidade (11) dessas publicações. O outro artigo $^{22}$ foi publicado em revista da Faculdade de Economia, Administração e Contabilidade da USP de Ribeirão Preto.

Os diversos temas tratados pelos Suplementos 1998 e 2003 foram abordados por essas publicações. Além de artigos que analisaram a diversidade de variáveis disponíveis sobre morbidade, acesso, planos de saúde, utilização de serviços de saúde e gastos privados, encontraram-se estudos que concentraram a sua análise em aspectos mais específicos, como a auto-avaliação do estado de saúde, as doenças crônicas, a incapacidade funcional física, o acesso a serviços odontológicos e a exames preventivos, a procura por serviços e o uso de serviços hospitalares, entre outros. Vários estudos analisaram subgrupos populacionais, em particular os idosos. Apesar de predominarem os estudos ao nível do indivíduo, encontram-se estudos que incorporaram o âmbito da família.

As desigualdades sociais e geográficas em saúde constituíram o principal aspecto avaliado nas publicações que empregaram dados dos Suplementos Saúde da PNAD. A despeito da diversidade de variáveis disponíveis no corpo básico do questionário, cabe, no entanto, ressaltar que essas ainda não foram exploradas em toda a sua potencialidade. Como exemplo, podese citar os dados sobre migração, que não foram objeto de análise em nenhum estudo sobre saúde e consumo de serviços de saúde selecionado nessa revisão.

Destaca-se a presença de estudos de economia da saúde que empregaram dados dos Suplementos Saúde para estudar a regulação do setor de planos de saúde no Brasil, o mercado e a tipologia de planos privados ${ }^{17,23-25}$, a existência de seleção adversa nesse mercado ${ }^{26}$, e as fontes de financiamento do consumo de serviços de saúde ${ }^{27}$.

Do ponto de vista do público-alvo dessas publicações, a maioria dos textos foi 
publicada por revistas científicas da Saúde Coletiva. No entanto, encontraram-se textos publicados por revistas da área de Demografia, Economia e Medicina. No âmbito governamental o IBGE, o IPEA e o Ministério da Fazenda publicaram estudos com base nos dados dos Suplementos Saúde. Porém, surpreendentemente, não foram encontrados estudos publicados pelo Ministério da Saúde e Secretarias de Saúde.

\section{O futuro da PNAD}

A tendência internacional para atender às demandas de informação dos órgãos governamentais e da sociedade é a revisão, pelas agências federais de estatística, dos sistemas atuais de condução de inquéritos, a fim de torná-los mais custo-eficientes. Em agosto de 2006, no II Encontro Nacional de Produtores e Usuários de Informações Sociais, Econômicas e Territoriais, o Instituto Brasileiro de Geografia e Estatística (IBGE) apresentou a proposta de um Sistema Integrado de Pesquisas Domiciliares Amostrais (SIPD). Esse projeto possibilita tanto a continuidade das pesquisas domiciliares amostrais existentes quanto a investigação de novos temas, o aumento da periodicidade de temas já pesquisados, a inclusão de investigações longitudinais e um maior detalhamento geográfico dos resultados.

A proposta do IBGE guarda similaridades com o Inquérito Domiciliar Integrado (Integrated Household Survey - IHS) do Office for National Statistics (ONS), órgão do governo britânico. Esse inquérito integra os conceitos, sistemas e amostras dos inquéritos referentes às pesquisas sobre trabalho (Labour Force Survey), domicílios (General Household Survey), gastos e alimentação (Expenditure and Food Survey), e temas diversos incluídos no inquérito omnibus (Omnibus Survey). Segundo seus idealizadores, essa integração objetiva a otimização dos recursos, a melhoria da informação, o aumento da consistência e da precisão das estatísticas oficiais, e uma maior flexibilidade e valorização dos produtos estatísticos ${ }^{28}$.

No tocante à otimização dos recursos, a criação de uma equipe única de entrevistadores que administram um questionário modular elimina a duplicidade de esforços gerados pela manutenção de equipes de campo, instrumentos e processos separados. O processamento e a produção de produtos de uma única fonte garantem uma maior economia de escala dos produtos estatísticos. Um maior valor desses produtos é obtido pela flexibilidade que as pesquisas integradas têm para acomodar outros inquéritos em estágios posteriores e a ampliação das oportunidades para análises entre as questões do módulo central e dos módulos tópicos garantem maior valor aos produtos estatísticos.

A proposta de integração dos inquéritos do ONS criou a necessidade de harmonização* das perguntas, dos conceitos e das classificações dos inquéritos integrados, a fim de obter uma maior coerência e consistência das estatísticas oficiais. $\mathrm{O}$ aumento na precisão das estimativas deriva da ampliação do tamanho da amostra anual para as variáveis do módulo central comum do questionário, o desenho amostral sem conglomerados e o aprimoramento da metodologia de ponderação. Além disso, o uso, entre os censos decenais, de variáveis sociais e econômicas (p.ex., renda e raça/cor) atende os propósitos políticos e propicia maior relevância às informações.

A amostra do IHS é obtida da soma de todos os endereços amostrados nos inquéritos separados. O questionário do IHS é composto por um módulo central comum e módulos tópicos. O módulo central comum contém perguntas fixas, mantidas no questionário por no mínimo 5 anos, e rotativas, mantidas durante 3 anos, relativas a variáveis sociodemográficas do tipo

A harmonização terminológica é o processo de alinhamento de termos e de definições entre línguas ou variantes de uma mesma língua (em relação à especificação de termos e de definições). A harmonização resulta muito mais de uma recomendação do que da obrigação de uma norma) 
censitárias, que são aplicadas em toda a amostra. Os módulos tópicos contemplam todos os temas abordados nos inquéritos atuais e outros temas relevantes para grupos específicos, que são aplicados apenas em partes da amostra.

A elaboração de um questionário relativamente curto e direto para uso nas pesquisas integradas do ONS baseou-se nos seguintes critérios para inclusão das perguntas: conter variáveis classificatórias e essenciais para análise, gerar relatórios que requerem altos níveis de precisão nacional/regional, serem adequadas tanto para a entrevista pessoal/presencial ou telefônica, que possam ser respondidas por respondentes substitutos ("proxy") e que não afetam a resposta geral ao questionário.

A fim de tornar pública a proposta do IHS e, desse modo, obter subsídios dos usuários dos seus produtos, o Office for National Statistics realizou uma consulta pública ${ }^{29}$. As preocupações relatadas pelos usuários das estatísticas britânicas diziam respeito tanto aos aspectos técnicos quanto às questões relacionadas à manutenção e integridade das séries históricas no novo modelo de inquérito. Esses usuários apontaram possíveis mudanças nas taxas e padrões das respostas, em decorrência da saturação da amostra; percepção que o inquérito integrado levaria a um número maior de entrevistas ou a entrevistas mais longas; aumento das chances de ser selecionado para entrevista; ou o fato de que mais pessoas seriam entrevistadas repetidas vezes, levando à fadiga do respondente $^{30}$. O uso de novas ferramentas e sistemas no processamento dos dados, e os atrasos no seu desenvolvimento e implantação também foram mencionados como fatores de comprometimento da qualidade dos dados. No tocante à descontinuidade das saídas e perda da integridade das séries históricas, isso poderia se dar pela mudança das perguntas, da sua ordem e dos métodos de ponderação; uso de novos sistemas; diminuição das taxas de resposta; e perda da qualidade do entrevistador. Como não havia garantias de melhoria da qualidade da informação com essas descontinuidades, os usuários do ONS sugeriram que se dispusesse de mais informações para avaliar os efeitos provocados pelas mudanças nos questionários.

No caso da proposta brasileira, o IBGE prevê a integração da Pesquisa Nacional por Amostra de Domicílios (PNAD) à Pesquisa Mensal de Empregos (PME), em uma investigação contínua com produção de resultados trimestrais sobre mercado de trabalho e rendimento. Na pesquisa integrada, o questionário terá um núcleo básico contendo as variáveis que serão respondidas por todos os entrevistados. A esse núcleo básico se associará um conjunto de questões sobre temas que podem ser investigados em um trimestre específico ou distribuídos nos trimestres. No entanto, os blocos sobre o mercado de trabalho serão sempre investigados. Alguns temas do corpo básico atual, tais como a educação e a fecundidade, teriam periodicidades a serem definidas.

A abrangência geográfica deverá ser no âmbito do país, grandes regiões, unidades da federação e regiões metropolitanas, que incluem municípios das capitais e áreas urbana e rural. A construção de uma amostra mestra (AM) servirá de infra-estrutura amostral para a condução de todas as pesquisas do SIPD do IBGE. Uma amostra mestra corresponde a um conjunto de unidades de área selecionadas de um cadastro, segundo um método probabilístico de seleção, de forma que seja possível selecionar subamostras para as diversas pesquisas.

O desenvolvimento de um marco amostral mestre possibilita maior custo-eficiência, qualidade e simplicidade aos inquéritos $^{31}$. Uma AM proporciona economia de custos ao compartilhar a preparação e atualização dos mapas e marcos subamostrais, selecionar de maneira rápida e fácil as unidades amostrais dos inquéritos integrados e inquéritos ad hoc, e empregar entrevistadores que residem próximo aos domicílios selecionados pela pesquisa. A utilização dos mesmos entrevistadores para vários inquéritos reduz o tempo para iniciar os inquéritos no campo e localizar os entre- 
vistados. Ademais, o uso da AM tem o potencial de acumulação dos temas, a expansão dos dados e a integração dos microdados através da sobreposição das amostras de dois ou mais inquéritos.

Entretanto, a AM apresenta limitações decorrentes das diferentes necessidades de inquéritos e da reutilização de unidades amostrais, especialmente no nível domiciliar. Essa reutilização oferece riscos de vieses decorrentes do aumento da não-resposta. Além disso, a qualidade das amostras mestras deteriora-se ao longo do tempo, devido às mudanças populacionais que ocorrem em taxas variadas em diferentes áreas geográficas. Desse modo, as medidas de tamanho da população ou do número de domicílios usadas para a seleção da AM deixarão de refletir a distribuição relativa da população do inquérito e levarão a um aumento dos erros amostrais das estimativas. As mudanças nas fronteiras $\mathrm{e}$ nas classificações administrativas também podem influenciar a AM, tornando a estratificação desatualizada. Em geral, a qualidade de uma AM depende de planejamento e manutenção adequados do seu marco amostral, podendo servir ao futuro censo populacional.

No SIPD, o esquema de estratificação levará em consideração a divisão administrativa e geográfica, a situação rural-urbana e a renda. As unidades de área e as unidades amostrais da AM correspondem aos setores censitários e aos domicílios, respectivamente. Somente os setores comum, especial de aglomerado subnormal e especial de aldeias indígenas serão incluídos na AM. O número de setores na amostra será aproximadamente $30 \%$ maior que o número atualmente selecionado na PNAD e PME. Isso confere maior espalhamento da amostra, permitindo a divulgação de resultados em domínios geográficos menores e a melhoria na precisão das estimativas.

O compartilhamento dos setores em todas as pesquisas diminui o custo provocado pelo aumento da amostra. O esquema de rotação de setores na AM produz a substituição de $2,5 \%$ dos setores a cada tri- mestre e a sua troca completa ao final de 10 anos. O número de domicílios visitados em um ano na pesquisa integrada será $15 \%$ maior do que o conjunto de domicílios visitado atualmente pela PNAD e PME. O número de visitas será o mesmo ao longo do ano, ao invés de as visitas estarem concentradas no último trimestre, quando coincidem os trabalhos de campo das duas pesquisas. O esquema de rotação de domicílios a ser adotado é o 1-2 (5), ou seja, um domicílio é pesquisado uma vez no mês, não participa da pesquisa nos dois meses seguintes e volta a ser pesquisado no $4^{\circ}$ mês, repetindo esse esquema cinco vezes ao todo. Em trimestres, isto significa que o domicílio é pesquisado uma vez no trimestre e permanece na pesquisa por cinco trimestres consecutivos, com 2 meses de intervalo entre as entrevistas. A sobreposição de domicílios é de $80 \%$ no trimestre e $20 \%$ no ano. A pesquisa integrada permite a acumulação de amostras para períodos maiores que um ano e a estimação em áreas geográficas menores.

Além das vantagens citadas, tais como a partilha entre as pesquisas do custo de seleção, melhor conhecimento da área, análise mais rica dos resultados, facilidades para investigação e maior distribuição geográfica das pesquisas, a pesquisa integrada possibilita que os respondentes sejam vinculados ao longo dos trimestres, constituindo, desse modo, bases de dados para estudos longitudinais.

Como apontado no caso do IHS do governo britânico, a proposta da pesquisa integrada PNAD-PME também impactará a continuidade e periodicidade das séries históricas, e a abrangência dos temas selecionados. Desse modo, será importante gerar informações que possibilitem avaliar a sua magnitude e se essas mudanças resultaram na perda de comparabilidade com os dados existentes. Outro desafio desse novo modelo de inquérito integrado será atender a todas as solicitações dos usuários, mantendo o tamanho dos módulos curto e manejável. Para atender essas demandas, o IBGE pretende realizar as pesquisas com 
a PNAD contínua em paralelo com as séries atuais da PNAD e da PME ao longo de 2 anos e realizar fóruns anuais e reuniões com gestores públicos de áreas específicas, produtores e analistas para discutir as mudanças a serem realizadas na pesquisa integrada, incluindo os temas específicos.

\section{Considerações finais}

As mudanças socioeconômicas, demográficas e tecnológicas e suas implicações nas políticas públicas exigem que os órgãos do governo produzam informações diversificadas, consistentes, comparáveis, atualizadas e oportunas à sociedade.

A geração de informação de base populacional e de âmbito nacional é essencial ao processo de planejamento e ao acompanhamento pela sociedade do cumprimento dos princípios constitucionais da saúde, como direito ao acesso integral e igualitário aos serviços de saúde. Dessa forma, a realização periódica de inquéritos domiciliares sobre saúde e consumo de serviços de saúde constitui um segmento importante da política de geração regular de informação domiciliar de base populacional do Ministério da Saúde.

Ademais, a proposta de incorporação de suplementos à PNAD, presente desde a sua concepção, possibilitou, ao longo dos anos, a geração de informações sobre diferentes temas de interesse das políticas governamentais a um custo acessível. Essa alternativa tem a vantagem de permitir a associação dos dados pesquisados nos suplementos às informações sobre as características dos domicílios e das famílias e aos aspectos sócio-econômicos e demográficos dos indivíduos, coletados anual- mente no questionário da PNAD.

No tocante à série histórica de Suplementos Saúde na PNAD, é importante ressaltar que ela tem possibilitado acompanhar aspectos relevantes da situação de saúde da população brasileira, bem como conhecer e monitorar, em diferentes recortes geográficos e socioeconômicos, as políticas de saúde voltadas para o acesso e a utilização de serviços de saúde. Outra vantagem da série histórica é permitir mapear as características e as mudanças nos mercados de serviços de saúde e suas interrelações, caracterizando as demandas e os padrões de utilização de serviços.

Além disso, os Suplementos Saúde compõem o conjunto de dados disseminados pelo IBGE, o que amplia a possibilidade de incorporação das informações de saúde pela sociedade e por diferentes áreas do governo, aspecto importante para o fortalecimento de ações intersetoriais, que têm que pautar as políticas voltadas para a melhoria da saúde do conjunto da população brasileira. Dessa forma, as futuras mudanças a serem implantadas pelo IBGE, com a criação do SIPD, devem ser analisadas na perspectiva das novas alternativas que estarão disponíveis à produção de informação domiciliar sobre saúde e consumo de serviços de saúde.

No âmbito do Ministério da Saúde, é importante mais ação, tanto na institucionalização do processo de geração de dados de base populacional como no uso regular dessa informação para o monitoramento das políticas setoriais. Cabe também ao Ministério o papel de disseminar e fomentar o emprego de dados populacionais pelas esferas estaduais e municipais no planejamento das políticas públicas de saúde.

\section{Referências}

1. Fletcher PR, Ribeiro, SC. A educação na estatística nacional. In: Sawyer DO (Org) PNADs em foco: anos 80. ABEP; 1988. p 13-32.

2. US Bureau of the Census, 1967. Atlântida: Un studio de caso en encuestas de hogares por muestra, Series ISPO, Washington, DC, $14 \mathrm{v}$.
3. IBGE. Disponível em: http://www.ibge.gov.br/home/ estatistica/populacao/trabalhoerendimento/pnad2003/ notas_brasil.pdf [Acessado em 28 de março de 2007].

4. NCHS - Americans assess their health: United States, 1987. Vital Health Stat Series 1990; 10(174). 
5. NCHS - Current Estimates From the National Health Interview Survey, 1994. Vital Health Stat 1995; 10(193).

6. Dachs JNW. Determinantes das desigualdades na autoavaliação do estado de saúde no Brasil: análise dos dados da PNAD/1998. Ciência \& Saúde Coletiva 2002; 7(4): 641-57.

7. Dachs JNW. Santos APR. Auto-avaliação do estado de saúde no Brasil: análise dos dados da PNAD/2003. Ciência \& Saúde Coletiva 2006; 11(4): 887-94.

8. Parahyba, MI e Simões CCS. A prevalência de incapacidade funcional em idosos no Brasil. Ciência \& Saúde Coletiva 2006; 11(4): 967-74.

9. Lambrew JM et allii - The effects of having a regular doctor on access to primary care. Med Care 1999; 34(2):138-51.

10. Instituto Nacional do Câncer. Inquérito Domiciliar sobre Comportamentos de Risco e Morbidade Referida de Doenças e Agravos não Transmissíveis. Ministério da Saúde; 2004

11. Silveira FG, Osório RG, Piola, SF. Os gastos das famílias com saúde. Ciência \& Saúde Coletiva 2002; 7(4): 719-31.

12. Becker RA, Lechtig A. Vacinação. In: Fundação Instituto Brasileiro de Geografia e Estatística (ed.). Perfil estatístico de crianças e mães no Brasil: situação de saúde 1981. Rio de Janeiro: Fundação Instituto Brasileiro de Geografia e Estatística; 1984. pp.175-207.

13. Oliveira LAP, Simões CCS. Morbilidade: níveis de percepção. In: Fundação Instituto Brasileiro de Geografia e Estatística (ed.). Perfil estatístico de crianças e mães no Brasil: situação de saúde 1981. Rio de Janeiro: Fundação Instituto Brasileiro de Geografia e Estatística; 1984. pp. 151-74.

14. Oliveira JC, Ferreira LMCR, Silva RMR. Aleitamento materno. In: Fundação Instituto Brasileiro de Geografia e Estatística (ed.). Perfil estatístico de crianças e mães no Brasil: situação de saúde 1981. Rio de Janeiro: Fundação Instituto Brasileiro de Geografia e Estatística; 1984. pp. 119-50.

15. Silva RMR. Atenção materno-infantil. In: Fundação Instituto Brasileiro de Geografia e Estatística (ed.). Perfil estatístico de crianças e mães no Brasil: situação de saúde 1981. Rio de Janeiro: Fundação Instituto Brasileiro de Geografia e Estatística; 1984. pp. 87-118.

16. Simões CCS, Oliveira LAP, Pereira NOM. A oferta e a utilização dos serviços de saúde. In: Fundação Instituto Brasileiro de Geografia e Estatística (ed.). Perfil estatístico de crianças e mães no Brasil: situação de saúde 1981. Rio de Janeiro: Fundação Instituto Brasileiro de Geografia e Estatística; 1984. pp. 65-86

17. Carvalho FM, Silvany Neto AM, Paim JS, Melo AMC, Azaro MGA. Morbidade referida e utilização de consulta médica em cindo populações do Estado da Bahia. Ciênc Cult 1988; 40(9): 853-8.

18. Médici AC, Campos MR. Brasil: Padrões de Morbidade e Utilização dos Serviços de Saúde (Uma análise da PNAD/ 86). Relatórios Técnicos. Rio de Janeiro: Escola Nacional de Ciências Estatísticas - Fundação IBGE; 1992.
19. Aquino EML, Menezes GMS, Amoedo MB. Gênero e saúde no Brasil: considerações a partir da Pesquisa Nacional por Amostra de Domicílios. Rev Saúde Pública 1992; 26(3): 195-202.

20. Kilsztajn S, Sugahara GTL, Lopes ES. Planos privados de assistência à saúde do idoso no Brasil. Rio de Janeiro; 2005 (IPEA - Coletânea Premiada).

21. Maia AC, Andrade MV, Ribeiro MM, Brito RJA. Estudo sobre a regulação do setor brasileiro de plano de saúde. Brasília; 2003 (Ministério da Fazenda - Documento de trabalho)

22. Santos MJ, Kassouf AL. Uma investigação dos determinantes socioeconômicos da depressão mental no Brasil com ênfase nos efeitos da educação. Econ Apl 2007; 11(1): 5-26.

23. Bahia L et al. Segmentação da demanda dos planos e seguros privados de saúde: uma análise da PNAD/98. Ciência \& Saúde Coletiva 2002; 7(4): 671-86.

24. Bahia L et al. O mercado de planos e seguros de saúde no Brasil: uma abordagem exploratória sobre a estratificação das demandas segundo a PNAD 2003. Ciência e Saúde Coletiva 2006; 11(4): 951-65.

25. Farias LO, Melamed C. Segmentação de mercados da assistência à saúde no Brasil. Ciência \& Saúde Coletiva 2003; 8(2): 585-98.

26. Alves SL. Estimando Seleção Adversa em Planos de Saúde. Revista Economia 2004. Dezembro.

27. Porto SM, Santos IS, Ugá MAD. A utilização de serviços de saúde por sistema de financiamento. Ciência \& Saúde Coletiva 2006; 11(4): 895-910.

28. Office for National Statistics. Consultation paper. Proposals for a Continuous Population Survey, July 2004. Disponível em http:/ /www.statistics.gov.uk/about/ consultations/ons_consultations/downloads/ CPS_Consultation.pdf (Acessado em 10 de junho de 2007.)

29. Office for National Statistics. Proposals for an Integrated Household Survey (Continuous Population Survey). Users' comments on the proposed design and progress report, October 2005. Disponível em http:// www.statistics.gov.uk/about/Consultations/ ONS_consultations/downloads/IHSConsultResponse.pdf (Acessado em 10 de junho de 2007).

30. Pettersson H. Design of master sampling frames and master samples for household surveys in developing countries. In: United Nations Statistics Division (org.) Household surveys in developing and transition countries. New York: United Nations; 2005.

31. Silva NN, Cunha TN, Quintanilha JA. Amostra mestra e geoprocessamento: tecnologias para inquéritos domiciliares. Rev Saúde Pública 2003; 37(4): 494-502. 